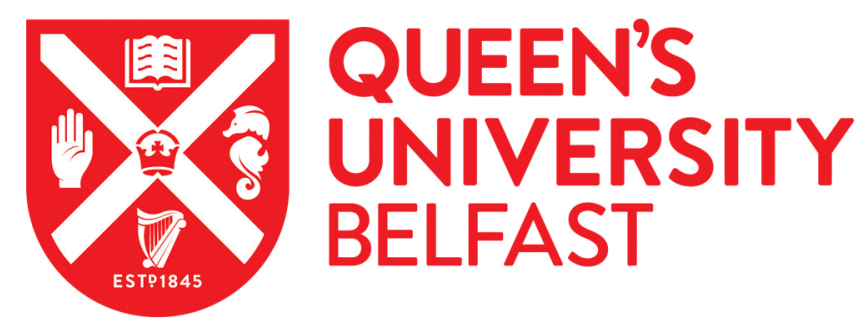

\title{
Automatic vetting of planet candidates from ground based surveys: Machine learning with NGTS
}

Armstrong, D. J., Günther, M. N., McCormac, J., Smith, A. M. S., Bayliss, D., Bouchy, F., Burleigh, M. R., Casewell, S., Eigmüller, P., Gillen, E., Goad, M. R., Hodgkin, S. T., Jenkins, J. S., Louden, T., Metrailler, L., Pollacco, D., Poppenhaeger, K., Queloz, D., Raynard, L., ... Wheatley, P. J. (2018). Automatic vetting of planet candidates from ground based surveys: Machine learning with NGTS. Monthly Notices of the Royal Astronomical Society, Advance Access. https://doi.org/10.1093/mnras/sty1313

Published in:

Monthly Notices of the Royal Astronomical Society, Advance Access

Document Version:

Peer reviewed version

Queen's University Belfast - Research Portal:

Link to publication record in Queen's University Belfast Research Portal

Publisher rights

(c) 2018 The Author(s) Published by Oxford University Press on behalf of the Royal Astronomical Society. This work is made available online in accordance with the publisher's policies. Please refer to any applicable terms of use of the publisher.

General rights

Copyright for the publications made accessible via the Queen's University Belfast Research Portal is retained by the author(s) and / or other copyright owners and it is a condition of accessing these publications that users recognise and abide by the legal requirements associated with these rights.

Take down policy

The Research Portal is Queen's institutional repository that provides access to Queen's research output. Every effort has been made to ensure that content in the Research Portal does not infringe any person's rights, or applicable UK laws. If you discover content in the Research Portal that you believe breaches copyright or violates any law, please contact openaccess@qub.ac.uk. 


\title{
Automatic vetting of planet candidates from ground based surveys: Machine learning with NGTS
}

\author{
David. J. Armstrong, ${ }^{1,2 \star ~ M a x i m i l i a n ~ N . ~ G u ̈ n t h e r, ~}{ }^{3}$ James McCormac,,${ }^{1,2}$ \\ Alexis M. S. Smith, ${ }^{4}$ Daniel Bayliss, ${ }^{1,2}$ François Bouchy, ${ }^{5}$ Matthew R. Burleigh,${ }^{6}$ \\ Sarah Casewell, ${ }^{6}$ Philipp Eigmüller, ${ }^{4}$ Edward Gillen, ${ }^{3}$ Michael R. Goad, ${ }^{6}$ Si- \\ mon T. Hodgkin, ${ }^{7}$ James S. Jenkins, ${ }^{8,9}$ Tom Louden, ${ }^{1,2}$ Lionel Metrailler, ${ }^{5}$ \\ Don Pollacco, ${ }^{1,2}$ Katja Poppenhaeger, ${ }^{10}$ Didier Queloz, ${ }^{3}$ Liam Raynard, ${ }^{6}$ \\ Heike Rauer, ${ }^{4,11}$ Stéphane Udry, ${ }^{5}$ Simon. R. Walker, ${ }^{1}$ Christopher A. Watson, ${ }^{10}$ \\ Richard G. West, ${ }^{1,2}$ Peter J. Wheatley ${ }^{1,2}$ \\ ${ }^{1}$ University of Warwick, Department of Physics, Gibbet Hill Road, Coventry, CV4 7AL, UK \\ ${ }^{2}$ Centre for Exoplanets and Habitability, University of Warwick, Gibbet Hill Road, Coventry CV4 7AL, UK \\ ${ }^{3}$ Astrophysics Group, Cavendish Laboratory, J.J. Thomson Avenue, Cambridge CB3 OHE, UK \\ ${ }^{4}$ Institute of Planetary Research, German Aerospace Center, Rutherfordstrasse 2, 12489 Berlin, Germany \\ ${ }^{5}$ Observatoire de Genève, Université de Genève, 51 Ch. des Maillettes, 1290 Sauverny, Switzerland \\ ${ }^{6}$ Department of Physics and Astronomy, Leicester Institute of Space and Earth Observation, University of Leicester, LE1 7RH, UK \\ ${ }^{7}$ Institute of Astronomy, University of Cambridge, Madingley Rise, Cambridge CB3 0HA, UK \\ ${ }^{8}$ Departamento de Astronomia, Universidad de Chile, Casilla 36-D, Santiago, Chile \\ 9 Centro de Astrofísica y Tecnologías Afines (CATA), Casilla 36-D, Santiago, Chile. \\ ${ }^{10}$ Astrophysics Research Centre, School of Mathematics and Physics, Queen's University Belfast, BT7 1 NN Belfast, UK \\ ${ }^{11}$ Center for Astronomy and Astrophysics, TU Berlin, Hardenbergstr. 36, D-10623 Berlin, Germany
}

Accepted . Received

\begin{abstract}
State of the art exoplanet transit surveys are producing ever increasing quantities of data. To make the best use of this resource, in detecting interesting planetary systems or in determining accurate planetary population statistics, requires new automated methods. Here we describe a machine learning algorithm that forms an integral part of the pipeline for the NGTS transit survey, demonstrating the efficacy of machine learning in selecting planetary candidates from multi-night ground based survey data. Our method uses a combination of random forests and self-organising-maps to rank planetary candidates, achieving an AUC score of $97.6 \%$ in ranking 12368 injected planets against 27496 false positives in the NGTS data. We build on past examples by using injected transit signals to form a training set, a necessary development for applying similar methods to upcoming surveys. We also make the autovet code used to implement the algorithm publicly accessible. autovet is designed to perform machine learned vetting of planetary candidates, and can utilise a variety of methods. The apparent robustness of machine learning techniques, whether on space-based or the qualitatively different ground-based data, highlights their importance to future surveys such as TESS and PLATO and the need to better understand their advantages and pitfalls in an exoplanetary context.
\end{abstract}

Key words: planets and satellites: detection, planets and satellites: general, methods: data analysis, methods: statistical

\section{INTRODUCTION}

The detection of exoplanets through photometric observation of transits has driven the field in recent years. Progress has been made from hard fought discoveries of single giant 
planets to large scale surveys capable of finding thousands of Earth, Neptune and Jupiter sized planets (Charbonneau et al. 2000; Bouchy et al. 2005; Collier Cameron et al. 2007a; Bakos et al. 2007; Borucki et al. 2010; Morton et al. 2016; Thompson et al. 2017). Future progress will focus on a combination of single rare or interesting systems alongside large scale population studies highlighting trends in the distribution and occurrence of planets.

A typical search for transiting planets might follow the process of 1) detrending the data (e.g. Stumpe et al. 2012; Smith et al. 2012; Tamuz et al. 2005), 2) running a search algorithm for transiting planetary signals (Collier Cameron et al. 2006; Kovacs et al. 2002; Mislis et al. 2015; Pearson et al. 2018), 3) vetting the results to produce a candidate list (e.g. Kepler's Robovetter, Coughlin et al. 2016), and 4) following up or validating these candidates to find new planets (Díaz et al. 2014; Morton 2012; Santerne et al. 2015; Torres et al. 2015). Step 3, vetting, often involves significant human input, whether by 'eyeballing' each significant signal or by setting thresholds on a series of semi-automated tests. This is especially true when considering ground based surveys (Bakos et al. 2004, 2013; McCullough et al. 2005; Alonso et al. 2004; Pollacco et al. 2006; Pepper et al. 2007; Wheatley et al. 2017), which must typically deal with more complex window functions and atmospheric noise sources than a space-based survey. Methods have been published to automate vetting in the past, with McCauliff et al. (2015) applying random forests to classify Kepler candidates, Thompson et al. (2015) and Armstrong et al. (2017) clustering Kepler and $\mathrm{K} 2$ candidate lightcurves with similar shapes and using the results to classify candidates, and Shallue \& Vanderburg (2017) applying neural nets to the classification of Kepler TCEs into planet candidates or false positives. These methods aim to build towards a situation where the process of planet detection can be fully automated, with the long term goal of combining steps 3 and 4; although planet validation on a large scale has been performed (Crossfield et al. 2016; Morton et al. 2016), this relies on significant human input prior to and during the process, and there is the potential for problems if care is not taken on individual candidates (Cabrera et al. 2017; Shporer et al. 2017).

Automation is both desirable and necessary. Future surveys will produce quantities of lightcurves and candidates beyond the scope of most methods. The soon to be launched TESS satellite (Ricker et al. 2014) is expected to observe tens of thousands of planets and hundreds of thousands of false positives, among $10^{8}$ targets (Sullivan et al. 2015). Far more apparently significant signals will need rejecting, as a result of instrumental or stellar noise. Robustly automating the process will be necessary for dealing with this data quantity, and crucial for considering statistical properties of the planet population. Testing and removing human bias in the selection process is non-trivial, and repeatable automated methods allow for sensitivity testing and quantifiable debiasing.

The only machine learning work in the exoplanet field applied to ground-based data to date is Dittmann et al. (2017), who used a neural net to identify 'trigger' events from single images of MEarth data. In this paper we present a technique to rank planetary candidates from the NGTS survey (Wheatley et al. 2017), using ground-based data taken over several months for 46470 target objects, sub- ject to the usual weather and visibility constraints. As such, the window function and noise properties of the data are among the most complex to have machine learning applied in an exoplanetary context. The method builds on that trialled on Kepler in McCauliff et al. (2015), with different features targeting ground-based data, and the addition of a self-organising-map to characterise signal shape efficiently. We aim to improve the survey pipeline through automatic ranking and selection of candidates, while demonstrating the method's applicability to an increased quantity of data with complex noise sources, in preparation for future surveys. Here we focus on the vetting procedure (the step from significant signals to planetary candidates) but the method has the potential to be expanded to include validation (planetary candidates to validated planets) in future.

We draw established techniques from the machine learning field, utilising random forests (Breiman 2001) and selforganising-maps (Kohonen 1982). Each has been demonstrated in an astrophysical context, in the realm of classifying stellar variability (Eyer \& Blake 2005; Mahabal et al. 2008; Blomme et al. 2010; Debosscher et al. 2011; Brink et al. 2013; Nun et al. 2014; Richards et al. 2012; Brett et al. 2004; Masci et al. 2014; Farrell et al. 2015), quasars (Carrasco et al. 2015), redshifts (Carrasco Kind \& Brunner 2014), solar flares (Liu et al. 2017) and asteroids (Huang et al. 2017), to name but a few.

This paper is structured as follows. In Section 2 we describe the NGTS survey and the context of this method in the pipeline. In Section 3 we describe our training sets and testing strategy for the models. In Section 4 we describe our methodology and hyperparameter choices, followed by Results in Section 5, tests to guard against bias and overfitting in Section 6, and we conclude in Section 7.

\section{THE NGTS SURVEY}

\subsection{Overview}

NGTS is a facility dedicated to detecting super-Earth and greater sized planets, through providing photometry at extremely high precision from the ground. NGTS obtains photometry at $0.1 \%$ precision in 1 hour on stars brighter than 13th magnitude through a combination of focused design and extremely stable autoguiding (McCormac et al. 2013, 2017). With a red-sensitive filter, the mission is optimised for the detection of small planets around $\mathrm{K}$ and early $\mathrm{M}$ stars. In total, NGTS is predicted to find $\sim 300$ new exoplanets and $\sim 5600$ eclipsing binaries (Günther et al. 2017). The NGTS facility consists of $1220 \mathrm{~cm} \mathrm{f} / 2.8$ telescopes sited at Cerro Paranal, Chile, using back-illuminated deep-depletion CCD cameras. Data is taken using one of the telescopes for each field, with each telescope typically observing two fields per night. Fields are not typically reobserved after the end of an observing season. At the point of writing, 10s cadence lightcurves for 46470 targets for on average 500 hours each spread over 250 nights were available for development and testing. See Wheatley et al. (2017) for a full description of the facility, data collection strategy and photometry reduction. 


\subsection{Candidate Detection}

Potential planetary transit signals are searched for using an implementation of the BLS algorithm, named orion, which has been used for the majority of transit detections in the WASP project. The most significant five periodogram peaks are extracted for each target, with each peak assigned a rank from 1 to 5 ordered by the BLS signal strength. orion removes periods within $5 \%$ of $1 \mathrm{~d}$ or $2 \mathrm{~d}$ before selecting the top 5 peaks. Peak periods which occur on a large number of objects in the same field are then removed (see Section 3 for detail), including any remaining near further aliases of 1 day. This leads to up to 5 periodogram peaks being used as separate detected candidates for each observed target, with less if some were removed. The large majority of these peaks represent instrumental signatures, harmonics, or other nonplanetary signals.

\subsection{Context}

Typically, the strongest signal in each lightcurve would be checked by a team of researchers looking for plausible planetary transits, and the best signals passed on for further photometric or spectroscopic follow-up. This human eyeballing is time intensive and can lead to complex biases in the signals selected, particularly for shallow marginal candidates, while also ignoring secondary BLS peaks on a target. The aim of this paper is to take steps towards automating this process, using machine learning algorithms to initially rank the candidate signals seen by researchers in order of their likelihood to be a planet, and with the eventual goal of removing human selection from the process entirely. Similar techniques have shown demonstrable success using spacebased lightcurves; here we wish to extend the technique to the often more challenging ground-based data. The procedure implemented and tested here forms part of the data processing pipeline for NGTS, and is currently used to rank candidates before they are seen by researchers. After further testing and development ranking may extend to actual selection of targets for followup.

\section{TRAINING AND TEST SETS}

\subsection{Training}

'Supervised' machine learning techniques require a training set, a distribution of examples designed to teach the algorithm how certain classes of signal appear. In a mature survey, a set of already detected planets and false positives could be used. However, for practical use it is necessary to implement these algorithms at an earlier stage, where no such set exists for the survey in question. Planets detected using other facilities have different quantities and qualities of data available, and have survey dependent parameter distributions, complicating their use. As such we turn to synthetic, injected planets.

Initially, we must generate model parameters for the star and injected planet. We randomly select actually observed NGTS targets, limiting the stellar magnitude from 9-14. Stellar effective temperature is then estimated using the 2MASS J-H colour for these targets, following Collier Cameron et al. (2007b), through

$$
T_{\text {eff }}=-4369.5(J-H)+7188.2
$$

with stellar radius $R_{*}$ estimated from $T_{\text {eff }}$ through

$$
\begin{aligned}
\frac{R_{*}}{R_{\odot}} & =-3.925 \times 10^{-14}\left(T_{\text {eff }}\right)^{4}+8.3909 \times 10^{-10}\left(T_{\text {eff }}\right)^{3} \\
& -6.555 \times 10^{-6}\left(T_{\text {eff }}\right)^{2}+0.02245\left(T_{\text {eff }}\right)-27.9788
\end{aligned}
$$

and stellar mass $M_{*}$ estimated as

$$
\frac{M_{*}}{M_{\odot}}=\left(\frac{R_{*}}{R_{\odot}}\right)^{-0.8}
$$

These relations are valid for single main sequence stars with $4000 \mathrm{~K}<T_{\text {eff }}<7000 \mathrm{~K}$ with a scatter around the relation of order 100K (Collier Cameron et al. 2007b). Transit parameters are drawn as uniform in log radius ratio between 0.1 and $2 \%$, uniform in orbital period between 0.35 and $20 \mathrm{~d}$, and uniform in impact parameter, allowing for grazing transits. We inject transits into 6 observing fields, and verify later that this does not bias results on the other fields. Model transits are injected into NGTS lightcurves prior to detrending. As such any effects of the detrending pipeline should be incorporated in the final lightcurve. Modified lightcurves are then searched for transits using the same BLS implementation as for the normal survey, and only those lightcurves where the injected signal was detected are put forward for use in our training set. As such the distribution of synthetic transits is affected by the NGTS sensitivity profile, and only transits which we could plausibly detect are used for training.

A training set is similarly needed for false positives, signals which may be flagged by the BLS algorithm but are not planetary in origin. In this instance such signals can include both instrumental artefacts (data gaps, remnant trends, etc) and astrophysical false positives (e.g. contaminating eclipsing binaries). The ideal training set for this group is the real detected candidates themselves. By using these candidates, we have the exact distribution of signals produced by the algorithm. Of course, within this set are some real planets, which represent the overall targets of the survey. Hence, we are proceeding under the assumption that the large majority of flagged signals are not planetary in origin and as such overwhelm the true planets present.

To limit the computational resources required, for both sets we apply a cut, removing candidate peaks which a) match another peak's period on the same object to within $0.2 \%$ (removing all but the strongest peak) or b) match a period which shows an abnormal prevalence within an object's observed field, with greater than 5 other candidates showing a period within $0.2 \%$. For context, fields had a median of 2354 candidates after removal of matching peaks on the same object, with fields ranging between 756 and 5708 . The aim of the cuts is to remove both alias periods on integer days caused by the observing pattern, as well as systematic noise periods connected to a particular field. The combination of targets, detrending, data quality and window function for a field can lead to spurious BLS detections which are common to several stars. In the latest run of NGTS data on all observed fields, the period cuts reduce the number of candidates to study from 188588 to 34668 (noting that one 
target object can have up to 5 candidates). There is some risk in applying this cut, as real planets with orbital periods very close to integer days would typically be removed from all fields. An example is WASP-131b (Hellier et al. 2017), which lies in an NGTS field but was not observed for long enough to obtain a full transit. However, such planets are extremely hard to detect from the ground regardless due to observing window aliases. We further remove any candidate where any of our calculated features returned a null result (typically poor quality lightcurves with very high noise or few datapoints).

We use two versions of the NGTS pipeline during the development and testing phases. These are known as 'TEST18', which after the above cuts contains 20166 candidates and 11005 synthetic injections, and the more developed 'CYCLE1706', which again after cuts contains 27496 candidates and 12368 injections.

\section{$3.2 \quad$ Testing}

Typically, the development of a machine learning algorithm incorporates initial training on the training set, development of model parameters and other meta-choices using crossvalidation on this training set, and final testing on an independent, not before seen test set. We adapt this to our survey by performing initial training and development on one iteration of the NGTS pipeline (TEST18), using crossvalidation to test and verify feature selection and model parameters. We then test the models on a later version of the pipeline which became available during development (CYCLE1706). Note that in the end, models are trained using CYCLE1706 data and injections, and tested using crossvalidation - but no model parameter choices or developmental decisions are made at this point, and hence the effect will be the same as the standard method. We appreciate that this is an unorthodox method for ensuring testing validity, but closely matches how the models will be used in practice; pipeline versions are continuously updated, and at each stage the models will be re-trained and applied on the new pipeline data, without new model development. To reinforce our test, we also perform a more typical training-test set analysis where the models are trained on the bulk of CYCLE1706 data then tested using a single field of CYCLE1706 data not before seen by the classifier, which produced similar results although with more limited numbers. The scores from these tests are presented in Section 5 .

\section{METHODOLOGY}

\subsection{Choice of techniques}

A wide array of machine learning techniques have been explored in the literature, with popular methods including deep neural nets (LeCun et al. 2015; Shallue \& Vanderburg 2017) and a variety of ensemble classifiers including Random Forests (Breiman 2001, hereafter RFs). Here we opt to use a combination of RFs with an unsupervised method, the self-organising-map (Kohonen 1982, hereafter SOM). This combination has been demonstrated successfully in the past for classifying variable stars (Armstrong et al. 2016) and the separate methods have both been demonstrated in a transiting exoplanet context (McCauliff et al. 2015; Armstrong et al. 2017). RFs have the advantage of being robust to varying data gaps and durations (field-to-field for example), as they deal with features extracted from the lightcurve rather than the lightcurve itself. RFs are also naturally extendable to include additional information, such as GAIA derived stellar parameters in the future, and in principle can be applied directly to other surveys or datasets to search for planets, although experience suggests this is rarely simple for any method. Finally, RFs allow a degree of interpretability, in terms of showing which features have the most deterministic power; this is a useful property for finding areas of potential weakness and bias in our models, and understanding how potential candidates are viewed by the model.

\subsection{SOM}

A SOM is a form of unsupervised machine learning, not requiring any training set. A SOM will cluster groups of inputs based on their proximity to each other, defining that proximity as the Euclidean distance between input points. We give an overview of the technique here; for more detail we refer the reader to Armstrong et al. (2016) for the setup and training methodology and Armstrong et al. (2017) for application to exoplanets and extraction of the features used.

The SOM used here consists of a 20x20 grid of "pixels', with each pixel consisting of a template transit. The grid is known as the Kohonen layer. The pixel templates are initialised randomly; as the SOM is trained they are permuted to resemble different shapes in the inputs. To train the SOM we use binned phase-folded lightcurve shapes, using periods, epochs and transit durations output from the transit search. Phase-folded lightcurves were cut to a window covering three transit durations, centred on the candidate transit, then binned into 20 equal-width bins. As such each SOM pixel has 20 values associated with it. Bin values were the weighted mean of all datapoints in the bin, using inverse square flux errors as the weights. We then normalise each transit such that the bottom of the transit has flux value 0 , and the baseline value 1 , measured using the lowest and highest quarter of points respectively. As such depth and duration information is removed. We randomly downsampled the real candidates to match the synthetic injections such that 11005 examples were available for each class, to give balanced inputs.

The training process then proceeds as follows. The input parameters are the initial learning rate, $\alpha_{0}$, which influences the rate at which pixels in the Kohonen layer are adjusted, and the initial learning radius, $\sigma_{0}$, which affects the size of groups that emerge. Initially each pixel is randomised so that each of its 20 elements lies between 0 and 1, as our binned transits have been scaled to this range. For each of a series of iterations, each input is compared to the Kohonen layer. The best matching pixel in the layer is found, via minimising the Euclidean distance between the pixel elements and the input. Each element in each pixel in the layer is then updated according to the expression

$$
m_{x y, k, \text { new }}=\alpha e^{\frac{-d_{x y}^{2}}{2 \sigma^{2}}}\left(s_{k}-m_{x y, k, \text { old }}\right)
$$


where $m_{x y, k}$ is the value $m$ of the pixel at coordinates $x, y$ and element $k$ in the binned transit, $d_{x y}$ is the euclidean distance of that pixel from the best matching pixel in the layer, and $s_{k}$ is the kth element of the considered input transit. Note that distances are continued across the Kohonen layer boundaries, i.e. they are periodic. Once this has been performed for each phase curve, $\alpha$ and $\sigma$ are updated according to

$$
\begin{gathered}
\sigma=\sigma_{0} e^{\left(\frac{-i * \log (r)}{n_{\text {iter }}}\right)} \\
\alpha=\alpha_{0}\left(1-\frac{i}{n_{\text {iter }}}\right)
\end{gathered}
$$

where $i$ is the current iteration, and $r$ is the size of the largest dimension of the Kohonen layer. This is then repeated for $n_{\text {iter }}$ iterations.

It is possible to use different functional forms for the evolution of $\alpha$ and $\sigma$; typically a linear or exponential decay is used. Brett et al. (2004) found that the performance of the SOM was largely unimpeded by the choice of form or initial value, as long as the learning rate does not drop too quickly. We find satisfactory results for the expressions above and values of $\alpha_{0}=0.1$ and $\sigma_{0}=20$, using $n_{\text {iter }}=300$ iterations.

The resulting distribution of candidates in the trained SOM is shown in Figure 1, and example trained pixel templates in Figure 2. The degree of clustering shown demonstrates that the method is still powerful even on the very different candidates arising from a ground based survey, as compared to Kepler where it was previously tested. In this case, rather than separating planetary candidates from false positives such as blended eclipsing binaries, the SOM is separating planetary candidates from a wide range of noise sources, astrophysical and instrumental, presented by the data. The location of a given candidate on the SOM is converted into the statistic $\theta_{1}$, which is described in Armstrong et al. (2017). The distribution of $\theta_{1}$ for each class is shown in Figure 3, and as expected from the observed clustering proves a powerful diagnostic in its own right.

The code used to implement the SOM can be found at ${ }^{1}$, and is an extension of the SOM incorporated in the PyMVPA Python package (Hanke et al. 2009).

\subsection{Random Forest}

Random Forests (Breiman 2001) are a versatile and effective machine learning technique. A RF classifier uses an ensemble of Decision Trees to perform classifications, using a set of input features. In our case features are extracted from the lightcurves and described below. Each tree attempts to independently classify a given input. A RF uses multiple trees to reduce the variance and bias of the model, through providing each tree with only a random subset of the available features. Each tree makes a choice between the available classes for the input using the information available to it, and the final output 'probability' is the fraction of trees which decided on each class. RFs are a supervised method requiring a training set, and are extremely versatile, assigning probabilities when classifying and so allowing ranking of candidates.

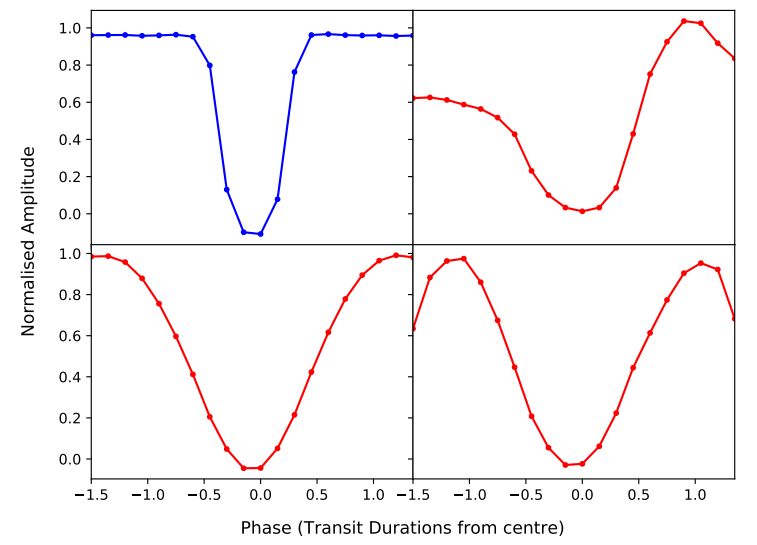

Figure 2. The SOM pixel templates for four key locations in Figure 1, one planetary (blue) and three non-planetary (red). Clockwise from top left the pixel indices are $[15,0],[16,11],[4,16],[1,16]$ ([x,y] format).

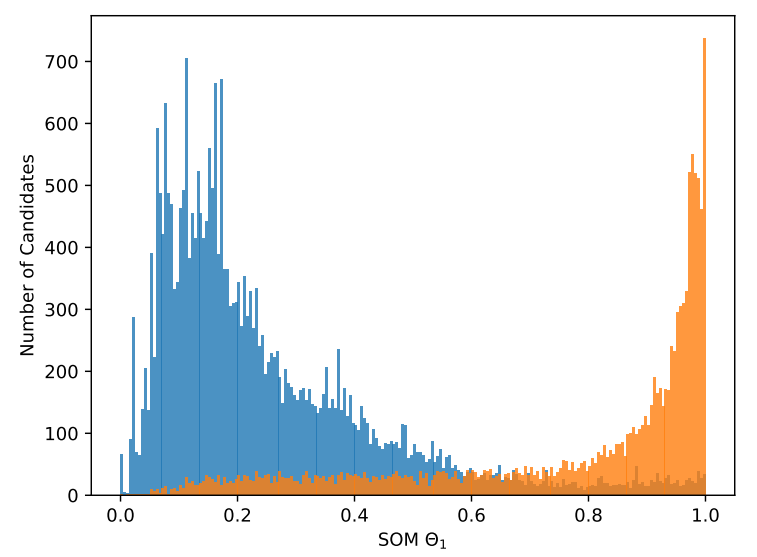

Figure 3. Distribution of $\theta_{1}$ for real candidates (blue) and synthetic transit injections (orange). The clustering evident in Figure 1 leads to distinct distributions in $\theta_{1}$.

RFs have been extensively discussed elsewhere, and we refer the reader to Richards et al. (2011, 2012); McCauliff et al. (2015); Armstrong et al. (2016) and multiple others for detail of their methodology, tree classification methods and examples of their use in an astronomical context. Our autovet code is a wrapper for the RF built into the scikit-learn module (Pedregosa et al. 2011). The code can be found at 2

\subsection{Features}

A Random Forest uses features passed to it to perform classifications. These features could be any relevant data, such as planet radius or the $\theta_{1}$ statistic discussed above. RFs are typically robust to uninformative features, meaning several 


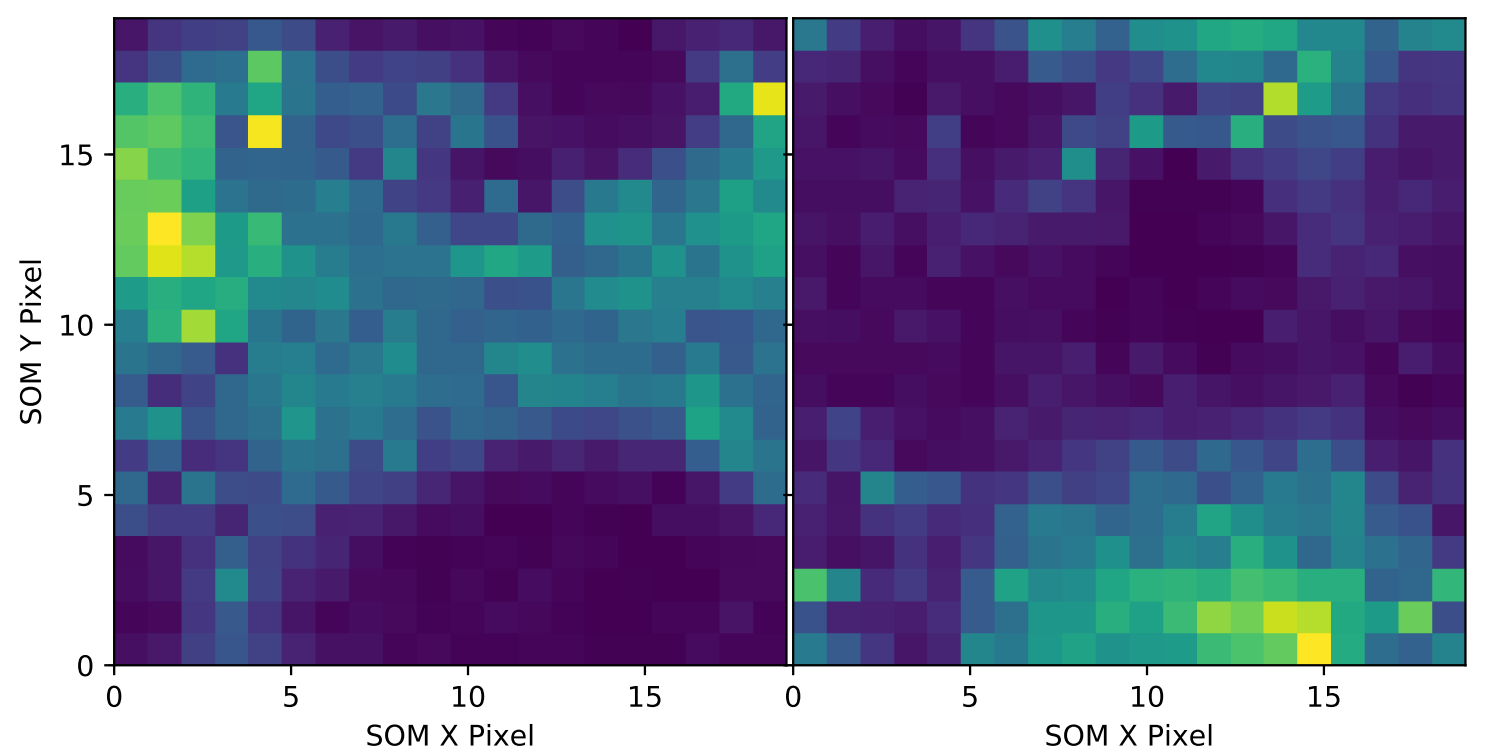

Figure 1. Histograms of positions on the trained 2 dimensional SOM for real candidates (left) and synthetic transit injections (right). Note the separation of the two groups.

can be trialled when building a classifier. We extract features based on the signal detection, the transit and fits to it, the overall phase folded lightcurve and several diagnostics designed to spot instrumental artefacts. The features we implement are shown in Table 1.

Initially we included several additional features incorporating information on noise inherent in the lightcurve: the root-mean-square, point-to-point percentiles, and median average deviation for example. During development it became clear that these features were surprisingly unhelpful; firstly, they only convey information indirectly, in that higher lightcurve noise only means a reduced chance of a planet for some transit and planet configurations. Secondly, as our synthetic injections were only injected into some fields due to computing constraints, using lightcurve noise related features biased the results to candidate lightcurves matching the distribution of noise in those fields. Fainter stars, more blended fields, and to an extent even poorly represented telescopes became downweighted, in a way that was hard to recover. We found that the simplest method to avoid these issues was to remove such features entirely. We show the effect this has on classifier performance in Section 5 .

There is a philosophical choice to make with regards to the features one makes available. One option is to incorporate knowledge of the known planet distribution; planets of a certain radius and orbital period are more likely than others, for example (Fressin et al. 2013). If trying to form a physically valid estimate of the likelihood that a given signal is a planet, then such an approach is likely necessary, but would require an accurate knowledge of the planet distribution, and care as to how this distribution was reflected in the output from the survey and synthetic transits used to train the model. Incorporating this information is somewhat analogous to adding a prior to the model.

However, when performing a blind search, incorporating knowledge of the 'known' planet distribution risks biasing the output rankings towards what you expect. This is particularly troublesome when considering new planet searches, as each survey has its own distribution of target stars and data characteristics, which will cause subtle or not-so-subtle effects in the observed planet distribution. Deliberately ignoring knowledge of some parameters, such as planetary radius, is then analogous to using an uninformative prior. The best approach is situation dependent and far from clear cut. Here in the context of a blind search using a new survey, we opt to exclude the planet radius and orbital period as direct parameters in an attempt to leave the resultant classifications as independent of prior knowledge as possible, and to avoid potential pitfalls resulting from incomplete information in our synthetic transit distribution. We do however include the directly fitted depth and duration of the transit signal, which while connected to the planet radius and orbital period provide strong constraints on eclipsing binaries.

Our injected transits are also limited by the parameter distributions used. In particular, the transit depths injected were between 0.1 and $2 \%$. Several planetary systems have transits larger than this depth, such as NGTS-1b (Bayliss et al. 2017), and are as such likely to be downweighted. Including transit depth in the model is desirable as it is a strong predictor for eclipsing binaries. We ameliorate this issue by proceeding with two parallel models, one including transit depth related features (WD-RF) and one without (ND-RF), which will be each be tested. The features shown in italics in Table 1 are the ones removed in the ND-RF model. The TransitSNR feature is included in both models, despite being related to the transit depth, as we found it was integral to model performance. Leaving TransitSNR in still allowed deep transits to be recovered in the ND-RF model during testing. 
Table 1. Features passed to the RF, ordered by feature importance calculated using the TEST18 data. A random feature was included for calibration, but not used in final classifications. Features in italics are dropped for the no-depth RF.

\begin{tabular}{|c|c|c|}
\hline Feature & Importance & Description \\
\hline SOM_ $\theta_{1}$ & 0.21593 & Transit shape statistic (Armstrong et al. 2017) \\
\hline TransitSNR & 0.13951 & Transit depth over standard deviation of out of transit lightcurve \\
\hline SOM_Distance & 0.10820 & Distance of transit shape to nearest SOM pixel \\
\hline Trapfit_t14phase & 0.06803 & Transit duration 1 st to 4 th contact points from trapezoid fit, phase units \\
\hline Trapfit_depth & 0.06652 & Depth of trapezoid fit, relative \\
\hline Fit_aovrstar & 0.06438 & Semi-major axis of transit fit, units of stellar radius \\
\hline RANK & 0.05769 & Rank of BLS detection on that candidate \\
\hline Even_Trapfit_depth & 0.03752 & As Trapfit_depth, even transits only \\
\hline Fit_rprstar & 0.03560 & Planet to star radius ratio from transit fit \\
\hline Odd_Trapfit_Depth & 0.03385 & As Trapfit_depth, odd transits only \\
\hline GAP_RATIO & 0.02847 & Fractional width of largest gap in phase-fold \\
\hline NPTS_TRANSIT & 0.02038 & Number of datapoints in transit \\
\hline Even_Fit_rprstar & 0.01529 & As Fit_rprstar, even transits only \\
\hline DELTA_CHISQ & 0.01502 & Difference in $\chi^{2}$ for BLS detection peak against baseline \\
\hline Odd_Fit_rprstar & 0.01236 & As Fit_rprstar, odd transits only \\
\hline NBOUND_IN_TRANS & 0.01054 & Number of night-boundaries within the transit box \\
\hline AMP_ELLIPSE & 0.00752 & Amplitude of ellipsoidal variation in the lightcurve \\
\hline MaxSecDepth & 0.00671 & Maximum depth of a secondary eclipse \\
\hline ntransits & 0.00589 & Number of observed transits \\
\hline MaxSecSig & 0.00567 & Maximum secondary eclipse significance \\
\hline SN_ELLIPSE & 0.00513 & SNR of ellipsoidal variation \\
\hline SN_ANTI & 0.00503 & SNR of strongest inverse transit detection \\
\hline Full_partial_tdurratio & 0.00429 & Ratio of Trapfit t14phase to Trapfit t23phase \\
\hline SDE & 0.00407 & BLS detection SDE \\
\hline PointDensity_ingress & 0.00373 & Density of points in ingress relative to average density in lightcurve \\
\hline PointDensity_transit & 0.00345 & Density of points in transit relative to average point density of lightcurve \\
\hline Even_Odd_trapdurratio & 0.00265 & Duration ratio (1st to 4 th contact) between trapezoid fits to even and odd transits \\
\hline Scatter_transit & 0.00201 & Standard deviation of points in transit relative to that of lightcurve \\
\hline Even_Full_partial_tdurratio & 0.00189 & Full_partial_tdurratio for even transits only \\
\hline MaxSecSelfSig & 0.00181 & Maximum secondary eclipse significance, normalised by other secondary detections \\
\hline Even_Odd_depthdiff_fractional & 0.00180 & Fractional depth difference between odd and even transit fits \\
\hline Even_Odd_trapdepthratio & 0.00178 & Depth ratio between trapezoid fits to even and odd transits \\
\hline Odd_Full_partial_tdurratio & 0.00132 & Full_partial_tdurratio for odd transits only \\
\hline Fit_depthSNR & 0.00116 & Depth from transit fit normalised by fit error \\
\hline Fit_chisq & 0.00115 & Best fitting $\chi^{2}$ of transit model \\
\hline Odd_Fit_depthSNR & 0.00096 & Fit_depthSNR for odd transits only \\
\hline missingDataFlag & 0.00091 & Fraction of missing data within 2.5 transit durations of transit \\
\hline Even_Odd_depthratio & 0.00087 & Depth ratio between fits to even and odd transits \\
\hline Even_Fit_depthSNR & 0.00087 & Fit_depthSNR for even transits only \\
\hline Random & 0.00066 & Random feature \\
\hline MaxSecPhase & 0.00063 & Phase of maximum detected secondary eclipse \\
\hline
\end{tabular}

\subsection{Transit Fitting}

Several of the features in Table 1 are derived from fits to the candidate signal, either to the whole lightcurve or to even or odd transits only. We perform two fits, one using a Mandel-Agol transit model (Mandel \& Agol 2002) implemented through the batman code (Kreidberg 2015) and one using a trapezoid.

Transit fit: Free parameters are the epoch, orbital period, semi-major axis over the stellar radius, and planet-star radius ratio. We fix the inclination at $\frac{\pi}{2}$, the eccentricity at 0 , and limb darkening to a quadratic law with parameters $[0.1,0.3]$. This is an intentionally oversimplified fit; our aim is to obtain approximations to what the parameters would be if the candidate was planetary in origin, even for extremely noisy signals which will often not be planetary or indeed astrophysical. As the method is intended to be used auto- matically on large datasets, no fits will be checked by eye, and hence only the most integral parameters can be fit for. The fit is performed using a least squares minimisation for simplicity. All features in Table 1 containing 'Fit_' derive from this method.

Trapezoid fit: Free parameters are the phase of transit, depth, time between 1st and 4th contact, and time between 2nd and 3rd contact. The orbital period is fixed. The fit is performed using a least squares minimisation for simplicity. All features in Table 1 containing 'Trapfit_' derive from this method.

\subsection{Feature Importance}

RFs allow estimation of the features contributing the most to the decision making process. Feature importance is estimated by considering where in the component Decision 
Trees features are considered - the higher up the tree, the more power a given feature has over the final classification. When averaged over the multiple Trees in a RF, this provides a ranking of features. Feature importances are shown in Table 1, where features are ordered by their importance to highlight the most significant.

To provide calibration, a feature consisting of randomly generated numbers was added to the training set, and its importance evaluated. The one feature with an importance less than this random feature was excluded from the final classifier.

\subsection{Centroid Information}

Due to its extremely precise autoguiding, NGTS is the first ground based telescope to routinely use shifts in the centroid position of a target during transit as a vetting method, achieving precision of 0.75 milli-pixel on average, and 0.25 milli-pixel in the best cases. The full description of this process is described in Günther et al. (2017). We trialled incorporating features marking significant centroid signals in the autovetter, but finally adopted a hybrid system whereby the centroid code independently flags certain targets. This is because certain forms of false positive, such as a blended transiting planet, might cause significant centroid shifts while still being interesting candidates that we may want to followup. Incorporating centroid related features in the RF would potentially downrank such candidates in a non-recoverable way. Furthermore, using centroid features would require simulating them for the synthetic transits, a somewhat arbitrary process involving significant human input in terms of the simulated distribution and thresholds used. When GAIA DR2 becomes available, it may be possible to incorporate centroid information in a sophisticated assessment of the probability of a given signal to lie on a given GAIA target, and this is planned in future development.

\subsection{Optimization/parameters}

The most important parameters defining the RF structure are:

- $n_{\text {est }}$ The number of decision trees. In general, more trees represent improved classification at the cost of more computer resources, with diminishing returns.

- $m f$ The maximum number of (randomly selected) features considered at each split within a tree. A typically good value is the square root of the number of features.

- $d$ The maximum depth (number of splits) each tree can have

- $m s$ The minimum number of samples required to split a node in the tree.

To optimize these parameters we use the TEST18 dataset. We perform a grid search over $n_{\text {est }}$ in [100,300, 500], $m f$ in $[2,3,5,6,7,9], d$ in $[2,5,8,11$, None], and $m s$ in $[2,3,4,5]$. For each combination, the out-of-bag (OOB) score was extracted from the RF. Each tree in the forest is fit using a random sample of the training data. The OOB score is the accuracy of the classifier on each input using only trees which were not trained using that input, and provides a quick estimation of the accuracy of the RF. The best OOB score (of $96.4 \%$ ) was found for $n_{\text {est }}=300, m f=5$, no $\max$ depth and $m s=3$. We adopt these values for $m f$ and $m s$, but apply a restriction on max depth, setting $d=8$. This is because although apparently better results are found for no limit on tree depth, a RF with no max depth is prone to overfitting, producing confident results in regions of parameter space not supported by the data. A larger depth supports more parameter space complexity, which is not always justified. The difference in OOB score is marginal, at $95.5 \%$.

As this classifier forms part of the NGTS pipeline, we put further effort into minimising the processing time required. With the above fixed parameters, we varied $n_{\text {est }}$, finding that significant gains in OOB score stop being made after an $n_{\text {est }}$ of 200. As such, we use 200 for our models. We do not expect this optimisation to change significantly through different pipeline versions, as while different versions will change the data quantity and specific detrending characteristics, the nature of the classification problem itself will not change.

\section{RESULTS}

\subsection{Classification Metrics}

With the form and parameters of the RF fixed, we turn to the CYCLE1706 dataset for testing. We retrain the two models with CYCLE1706, and use cross-validation to test their performance. Cross-validating consists of successively excluding blocks of inputs from the classifier, with the inputs in a random order. The classifier is then trained on the non-excluded data, and the trained classifier used to classify the initially excluded inputs. A new classifier is trained for each excluded block. In this way, classifications are obtained for each input using classifiers which were not trained on that input. The NGTS pipeline will continue to develop, and hence using CYCLE1706, a version which the RFs were not optimised on, is the ideal test for how they will perform in practice. It is necessary to retrain the models however, now and with each new pipeline version, to account for the varying dataspan and noise properties which different versions present.

Classification problems are typically measured using a series of standard metrics. Here we use three: the Precision (the fraction of signals that are classified as planets which are injected planets), the Recall (fraction of injected planets which are classified as planets), and the AUC (area under the receiver-operator characteristic curve, see Fawcett 2006). These results are shown in Table 2, for models with and without lightcurve noise related features (see Section 4.4) and with and without transit depth related features (see Section 4.4 and Table 1). The overall performance of the classifier is largely unaffected by these choices, showing a slight improvement as more features are added, but this masks divergent performance on specific subsets of candidates. In summary, noise related features introduce biases associated with specific fields and cameras used to generate the training set, and are hence removed from here on. Depth related features, when included, downrank several known planets with deep transits $(>2 \%)$, as this is the limit of our synthetic injections. Removing these features recovers these 
Table 2. Cross-validated classification scores for Random Forest models.

\begin{tabular}{llll}
\hline Features used & Precision & Recall & AUC \\
\hline No Depth, No Noise & 0.892 & 0.905 & 0.976 \\
With Depth, No Noise & 0.897 & 0.910 & 0.978 \\
$\quad$ Cross- Validation & 0.897 & 0.910 & 0.978 \\
$\quad$ Train- Test & 0.99 & 0.93 & 0.979 \\
No Depth, With Noise & 0.896 & 0.910 & 0.979 \\
With Depth, With Noise & 0.901 & 0.914 & 0.980 \\
\hline
\end{tabular}

Precision and recall calculated using a threshold of 0.5

planets, at the expense of greater weakness to eclipsing binaries. For the remainder of the paper, we explore models without noise related features, but with (WD-RF) and without (ND-RF) depth related features, as scientifically interesting planets such as NGTS-1b (Bayliss et al. 2017) can present deep transits.

For the WD-RF model, we show scores for both cross validation and for performance on an unseen test set, which consisted of one field of CYCLE1706 data not seen by the classifier. The test set consisted of 2251 synthetic injected planets and 348 real candidates, after period cuts were run as described in Section 3. The numbers are unbalanced as we used a single NGTS field with a limited number of candidates, leading to an anomalously high precision but otherwise comparable results. We proceed using the crossvalidation results, as they are similar if slightly lower and incorporate more of the available data to produce the values.

We note that the scores are not precisely correct, as their calculation assumes each training set is completely accurate. As some fraction (unknown as yet) of our real candidates will be real planets, this assumption does not hold. Given known planet occurrence rates and the hit rate of other ground based surveys we expect the proportion of real planets to be low enough to make little difference to the scores. In Günther et al. (2017), we previously estimated that $\sim 97 \%$ of all initial BLS detections in NGTS are caused by false positives (eclipsing binaries and background eclipsing binaries) and false alarms (systematic noise). This value is in line with the findings from other surveys. In comparison, initial detections in CoRoT and HAT contained 95-98\% of false signals (Almenara et al. 2009; Latham et al. 2009; Hartman et al. 2011).

The distribution of probabilities output by the WD$\mathrm{RF}$ classifier is shown in Figure 4, for the real candidates and synthetic injections. Probabilities are calculated using cross-validation, in which the candidates are randomly ordered, and successive blocks of 500 are removed. For each removed block, the classifier is trained on the remainder of the candidates, and then used to classify the withheld 500 . The process is repeated with the classifier retrained each time until all candidates have been classified. The efficacy of the classifier at separating the two classes is evident. A tail of real candidates with high probabilities of being in the 'Planet' class can be seen, and these represent potential planets discovered by the classifier. We note that there are two use cases for the classifier here: one to identify highpriority candidates, and the other to rank all the candidates.

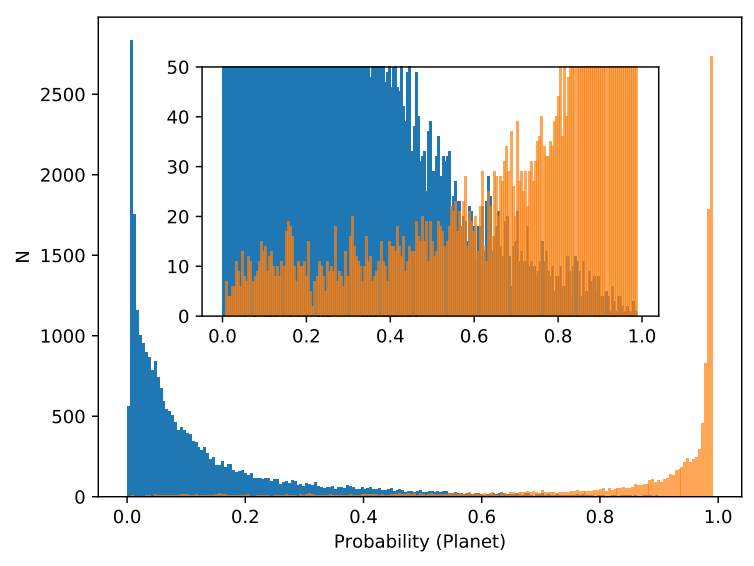

Figure 4. RF output planet probabilities for the real candidates (blue) and synthetic injected transits (orange). A zoomed in inset shows the few misclassified injections. Real candidates with high planet probabilities are strong candidates for further followup, unlike the majority of signals.

In the context of ranking, even a score of 0.2 puts a planet above $\sim 80 \%$ of the signals.

\subsection{Key Features}

Feature importances are calculated as described in Section 4.6. We list the top 10 features here for the ND-RF model, with a more detailed description than given in Table 1. Note that the feature importances listed in Table 1 are for the WD-RF model, which changes the order slightly.

- SOM_ $\theta_{1}$ Transit shape statistic, falling between 0 (for transits similar in shape to the set of false positives) and 1 (for transits similar in shape to the synthetic injections). The statistic is produced by comparing the binned transit to a SOM generated from the complete set of candidates and synthetic transits, and identifying the closest match. See Armstrong et al. (2017) for a full description, and Figure 3 for the calculated distributions.

- TransitSNR The signal-to-noise of the detected transit signal, measured on the transit duration. The phasefolded lightcurve is binned on $80 \%$ of the transit duration (to avoid ingress and egress). TransitSNR is the bin value of the in transit bin divided by the standard deviation of the out of transit bins. Note that this measures the 'detectability' of the transit. While the depth derived will not be accurate for highly V-shaped transits, TransitSNR provides a measure of how easily such transits will be seen by our BLS implementation. The distributions of TransitSNR are shown in Figure 5. We additionally show the distribution of transit depths as measured by the trapezoid fit in Figure 6 to show the difference between SNR and depth.

- SOM_Distance When a signal is compared to the SOM and finds its closest match, the distance between the signal and this match is calculated. This is typically higher for false positives (as these have a wider range of possible shapes), and so the distance can help to resolve the classification in cases marginal in SOM $\theta_{1}$. The distribution of SOM Distance is shown in Figure 7. 


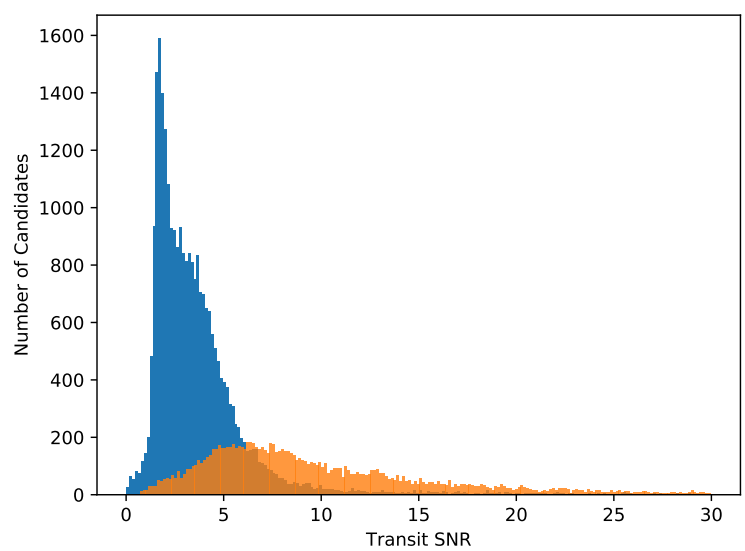

Figure 5. As Figure 3 for the TransitSNR feature.

- Trapfit_t14phase A trapezoid model is fitted to the detected signal (see Section 4.5). One of the outputs of this fit is the transit duration, in phase, between the 1st and 4th contact points.

- Fit_aovrstar We also use batman (Kreidberg 2015) to fit a Mandel-Agol transit model to the detected signal (see Section 4.5). One output from this fit is the putative planet's semi-major axis in units of the stellar radius.

- Rank Our BLS algorithm outputs the top 5 periodic signals for each candidate. The rank (an integer between 1 for the strongest and 5 for the weakest) represents the order for the signal under question.

- GAP_RATIO The fractional width of the largest gap in coverage in the phase-folded lightcurve.

- DELTA_CHISQ The difference in $\chi^{2}$ between a box model and a flat model for the peak BLS detection.

- MaxSecDepth The phase folded lightcurve is scanned for secondary eclipses, between phase 0.3 and 0.7 , using a box of width the transit duration. The most significant possible secondary eclipse in this region is found and the mean flux value in the eclipse extracted.

- AMP_ELLIPSE The amplitude of any ellipsoidal variation in the phase-folded lightcurve.

- NPTS_TRANSIT The number of data-points within the best fitting transit box.

\subsection{Known Planets}

Four published planets, 3 as yet unnamed confirmed NGTS discoveries and 42 previously flagged planetary candidates are available in the dataset for testing. These form ideal test cases for the algorithm. Results for the confirmed planets are shown in Table 3. For the ND-RF, all but one planet are detected with scores greater than 0.5 . From a ranking perspective, we note that even the lowest scoring planet, with a score of 0.27 , ranks in the top $15 \%$ of candidate signals, demonstrating the efficacy of the RF in aiding detection. For the WD-RF, the planets with transit depth larger than $2 \%$ are predictably downranked, in particular HATS-43b with a depth of $2.9 \%$.

The 42 flagged planetary candidates will contain some

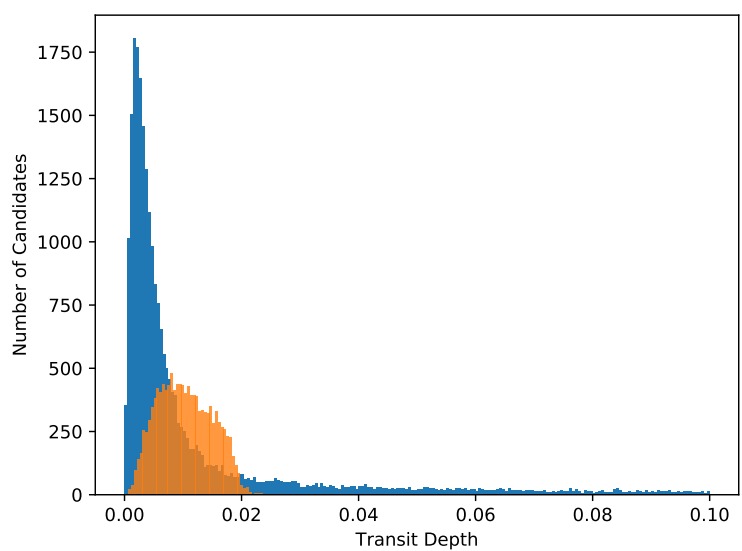

Figure 6. As Figure 3 for the Trapfit_depth feature. The histogram for real candidates (blue) extends to higher depths which are not shown for clarity.

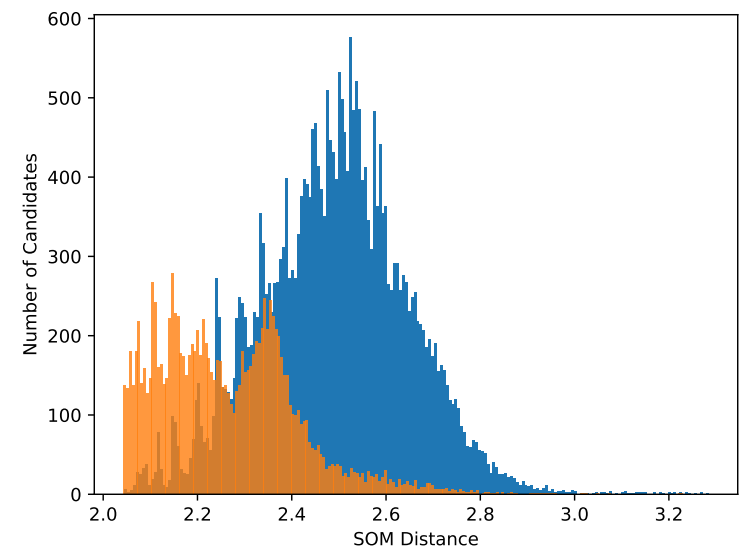

Figure 7. As Figure 3 for the SOM Distance feature.

real planets and some false positives. The cumulative frequency as a function of their RF output for these candidates is shown in Figure 8, along with that for the entire set of signals studied. It is clear that the RF ranks the 42 candidates much higher than the general background of signals, and more so for a subset of the candidates. We note that there is a bias in the sample studied here - as these are early results from the survey, and pre-NGTS planets arise from ground

Table 3. Scores for observed known planets

\begin{tabular}{lllr}
\hline Planet & ND-RF & WD-RF & Discovery Reference \\
\hline HATS-43b & 0.95 & 0.04 & Brahm et al. (2017) \\
WASP-98b & 0.51 & 0.11 & Hellier et al. (2014) \\
WASP-68b & 0.86 & 0.93 & Delrez et al. (2014) \\
NGTS-1b & 0.73 & 0.65 & Bayliss et al. (2017) \\
NGTS-xb & 0.27 & 0.39 & in preparation \\
NGTS-xb & 0.63 & 0.20 & in preparation \\
NGTS-xb & 0.93 & 0.95 & in preparation
\end{tabular}

$\mathrm{ND}-\mathrm{RF}=$ No depth features, WD-RF $=$ With depth features 


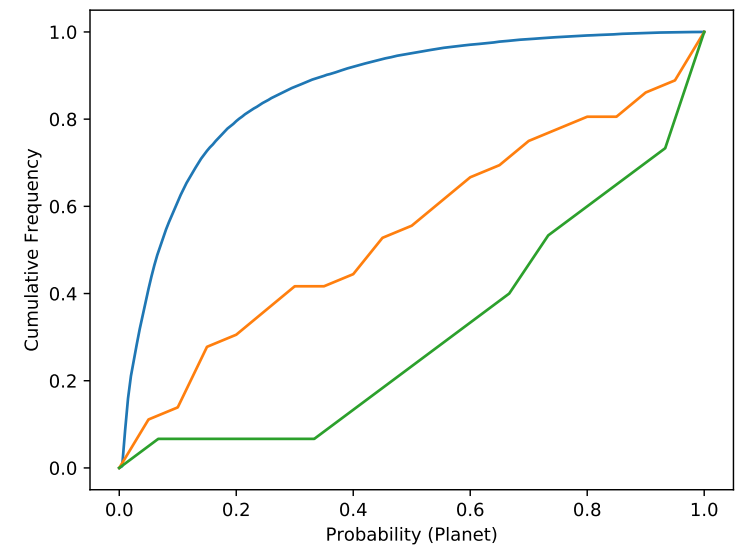

Figure 8. Normalised cumulative frequency of candidates for the total set of BLS signals, i.e. in the majority false positives (blue), for candidates already pre-selected by eye for follow-up (orange) and for the pre-selected candidates ignoring those deeper than $2 \%$ or $\mathrm{V}$-shaped (green). The difference of the orange and green curves to the blue shows the model's effectiveness at ranking good candidates higher than the background of signals.

based surveys, the sample of candidates and planets is typically larger, with deeper transits than we expect on average. Several of the highlighted candidates are shallower (in the Neptune radius regime) however, with promising follow-up work ongoing.

\subsection{Highly Ranked Candidates}

Both models highlight numerous candidates for further observations. The ND-RF flags 53 candidates with planet probability $>0.9$, and 1355 with probability $>0.5$, out of a total of 27498 that passed the period matching cuts. The WD-RF flags 50 and 1294 respectively. These are in the majority but not exclusively the strongest BLS signals detected on an object.

We cannot lay out all of these candidates here, but focus on the top 10 in each case to illustrate the power of the method. Five of these top 10 overlap between the ND-RF and WD-RF, demonstrating the robustness of the models. In the WD-RF, the top 10 contain 4 candidates now undergoing follow-up spectroscopic observations (1 newly flagged by this method), 3 candidates that were previously selected by eye which were revealed as double lined spectroscopic binaries from spectroscopy (none show obvious characteristics which would exclude them as planets from the lightcurve alone, demonstrating the importance of follow-up observations at this stage of development), 2 candidates which show clear interesting potentially planetary signals but which are blended with other targets in the photometric aperture, and one candidate which shows an interesting signal but which is likely a giant host star. These results are encouraging, with 7 out of 10 candidates worthy of further follow-up and the remainder understandable given the model inputs. These latter 3 cases demonstrate a weakness - at present no information is given regarding blending or host star type, and we plan to incorporate features with this information in future iterations. Such information will become readily available with the GAIA DR2 data release.

The additional 5 candidates highlighted by the ND-RF model show 2 candidates amenable to follow-up observations (one new, one previously scheduled), 2 strong but too deep candidates (4\% and 3\% transits, including one which is also blended in the aperture), and one interesting signal but on a likely giant host star.

\section{VALIDATION TESTS}

\subsection{Sensitivity}

An advantage of using a synthetic array of injected transits is that we can test the performance of the RF on a known dataset, investigating our sensitivity and providing some interpretation of how the RF is working. We note however that such a study can only take us so far - the distribution of real planets in the data may be different, especially outside the range of the synthetic injections, and hence the RF may respond differently. Without a sample of detected planets from the mission this is impossible to test directly, but early results on detected planets are promising (Section $5.3)$.

We begin by studying the parameters of the synthetic transits which were not recovered, using cross-validated probabilities in all cases and considering the WD-RF model. Figures 9 and 10 show the output planet probability of the transits as a function of their SNR, for synthetic injected transits and for real candidates respectively. The SNR shown here is measured as the depth of the binned transit, normalised by the standard deviation of out-of-transit bins of the same duration. The data shows that our sensitivity begins to drop at SNR of 7-8, interestingly in line with the threshold of 7.1 used by the Kepler pipeline (Thompson et al. 2017, and reference therein), although their calculation of SNR is different. Below this threshold the uncertainty on the model increases. While lower SNR planets are not always detected, the dispersion increases such that even at the lowest measured SNR some injected planets are detected at high confidence.

Having found the cut-off in sensitivity for SNR, we can investigate what it is that causes the RF to drop these planets. The most important feature used by the RF is the SOM $\theta_{1}$, and we plot the output planet probability as a function of this and the SNR in Figure 11. Low SOM $\theta_{1}$ and low SNR both cause a drop in planet probability. The cause of this is twofold; either low SNR transits are misinterpreted by the SOM as their shape is unclear, or grazing transits with a pronounced $\mathrm{V}$-shape, and hence low $\theta_{1}$, have naturally lower depth and hence low SNR. Figure 12 shows the output planet probability as a function of the transit depth and orbital period. Longer period and decreased depth are associated with lower planet probability as expected, as in each case the SNR will drop. However it is encouraging that for short periods where many transits are observed, there is little dependence on transit depth, showing that our models are not biased in this regard. The same is true for larger depths and longer periods. We note that as shown in Figure 9 , even the more extreme long period low depth transits are sometimes detected by the model. Finally Figure 13 shows 


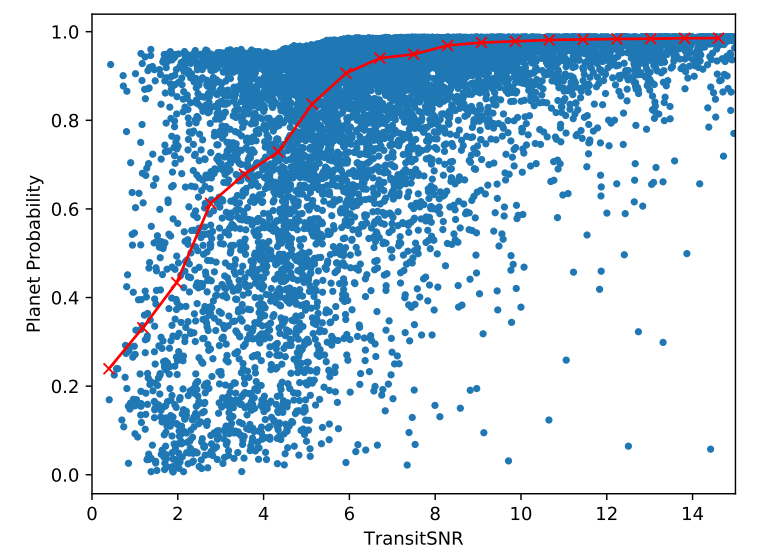

Figure 9. RF output planet probability as a function of transit SNR for the synthetic injected transits, with higher SNR cases not shown. The median planet probability for a series of bins is shown as red crosses.

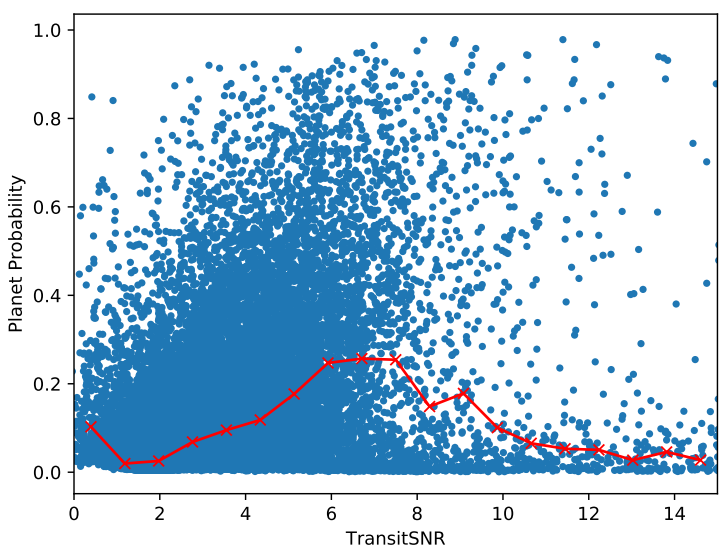

Figure 10. As Figure 9 for real candidates.

the effect of the SOM distance on synthetic transits. Transits only have a high distance when they are at low SNR and their shape is not well defined, highlighting that the criteria is not solely SNR but a combination of SNR and how the transit shape interacts with the noise in the lightcurve.

\subsection{Field Dependence}

As we injected transits into only a subset of observed fields, it is important to check that the RF is not biased towards candidates in these regions. This is potentially problematic, as fields are observed by different cameras, and can have differing noise properties, window functions, durations and crowding. We verify this by plotting the planet probability distribution for real candidates by field in Figure 14. If candidates from a field are downranked due to their membership in that field, the distribution from that field will be affected. The fields used for injections are highlighted, and are not divergent from the set.

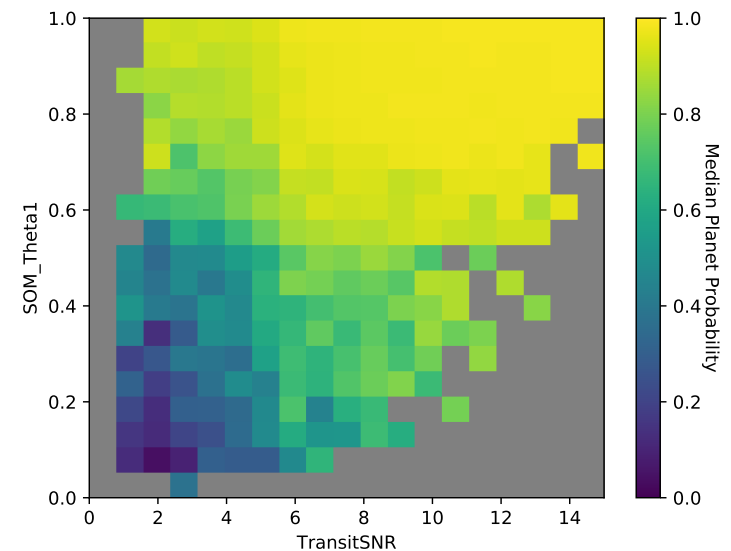

Figure 11. Median planet probability as a function of TransitSNR and SOM $\theta_{1}$ statistic, for the synthetic injected transits. Bins containing fewer than 5 candidates are in grey. Higher SNR cases are not shown.

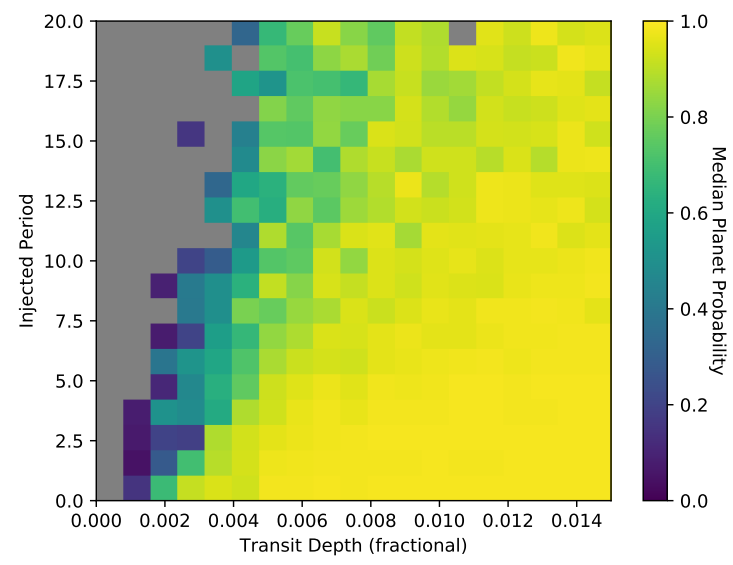

Figure 12. As Figure 11 for injected transit depth and orbital period. Bins containing fewer than 5 candidates are in grey. Larger depth cases are not shown.

\subsection{Outliers and Overfitting}

A common issue when applying machine learning techniques is overfitting; models can become confident about classifying regions of parameter space not supported by the data, especially when the model complexity is not justified by the quantity of training data. More problematically, if training data is sufficient but lacking in a particular region of parameter space, or outliers in the training set are present, problems can occur which may be harder to diagnose. This issue is particularly problematic where results on individual cases are important, as is the case in a survey searching for a relatively small number of planets. Overfitting may result in overconfident classifications, or erroneous outputs for specific objects. Such errors can be shown during testing, but if they occur in small regions of parameter space not covered by the test set then identifying the issue, or even that there was an issue, would be challenging.

Each machine learning method has options for guard- 


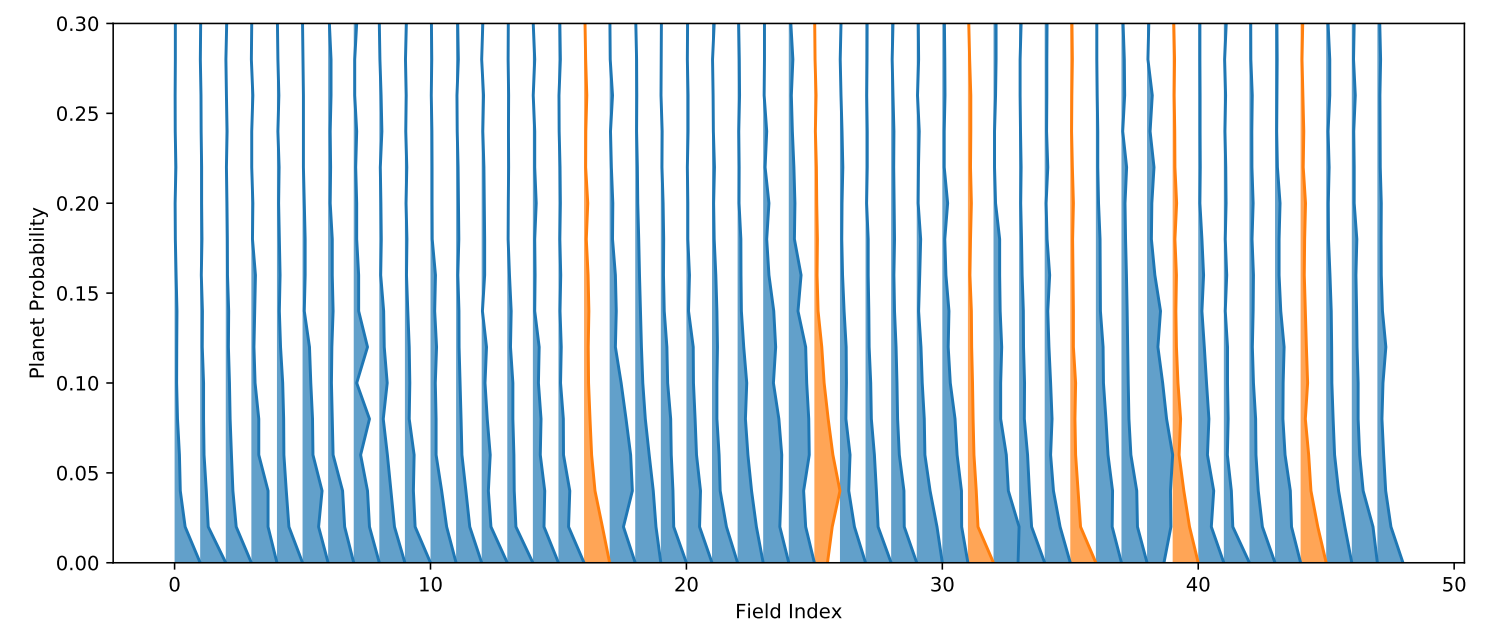

Figure 14. Planet probability distributions for the real candidates, as a function of observing field. Fields in orange are those where the synthetic transits were injected. Fields are ordered by median planet probability.

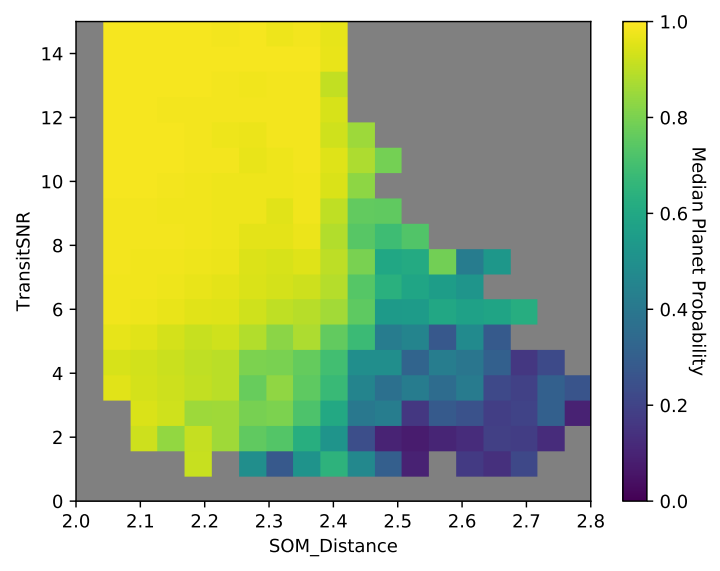

Figure 13. As Figure 11 for TransitSNR and SOM_Distance, for the synthetic injected transits. Bins containing fewer than 5 candidates are in grey. Higher SNR cases are not shown.

ing against overfitting. In the case of a RF, a key option is the maximum depth of the component decision trees. The depth is described in Section 4.8, and is the number of splits each tree is allowed to make. Reducing the maximum depth guards against overfitting, as it reduces the potential complexity of the RF. For example, when optimising our models with no maximum depth imposed, we obtained tree depths of 25-30. Imposing a maximum depth of 8 did not significantly change the accuracy of the models, and as such we conservatively adopted this limit. Another option would be to search for outliers (through for example an Isolation Forest, Liu et al. 2008) and remove these before training.

In this context, we have an injected synthetic distribution which by definition does not contain outliers (more accurately, any outliers in our specific parameter space arise because of peculiarities of the lightcurve rather than the planet itself, and hence will be rare). The real candidates will contain anomalous and unusual objects, but as these are classified as false positives in the training set they will only lead to the RF classifying other similarly unusual candidates as false positives, which in this context is desirable. Note that by anomalous here we describe unusual noise sources and combinations of features; such objects will not typically be scientifically interesting, and could in any case be targeted separately if desired. In essence, for the purposes of ranking candidates we do not consider outliers to be an issue here. However, should one need to trust the output planet probability for an individual candidate (for validation for example), the issue is much more important. In such a use case, subtle biases of the model must also be taken into account, and it is not yet clear how to adequately establish trust on individual inputs for often fundamentally uninterpretable machine learning methods. We leave that development for future work.

\section{CONCLUSION}

We have presented a method for ranking candidates from a transiting planet search. Through incorporating random forests and self-organising-maps we are able to obtain an AUC score of $97.6 \%$ on data from the NGTS survey, showing that such techniques are effective for ground based photometric data with complex window functions compared to the space-based photometry typically used as a test case. We demonstrate that machine learning methods can be effective for new and active surveys where a sizeable training set does not yet exist by utilising injected synthetic transits. Such simulations further allow a degree of testing and interpretability of the model.

Improvements to our method are possible, through inclusion of stellar data from the GAIA satellite, increasing the size of the training set, taking more account of outliers and the parameter space viewed by the model, and potentially by exploring alternate inherently probabilistic classifiers. In particular, understanding potential biases and improving re- 
liability on individual candidates is crucial for fully taking advantage of automated methods.

We also present a publicly accessible code, autovet, which can calculate features from lightcurves and act as a wrapper for various scikit-learn machine learning implementations. Similar techniques are becoming increasingly prevalent as surveys produce increasing quantities of data, and will be crucial in maximising the scientific return of missions such as TESS and PLATO.

\section{ACKNOWLEDGEMENTS}

This publication is based on data collected under the NGTS project at the ESO La Silla Paranal Observatory. The NGTS instrument and operations are funded by the consortium institutes and by the UK Science and Technology Facilities Council (STFC; project reference ST/M001962/1). DJA, DP, PJW and RGW are supported by an STFC consolidated grant (ST/P000495/1). MNG is supported by the UK Science and Technology Facilities Council (STFC) award reference 1490409 as well as the Isaac Newton Studentship. JSJ acknowledges support by FONDECYT grant 1161218 and partial support by CATA-Basal (PB06, CONICYT) We thank the anonymous referee for helpful comments which improved the manuscript.

\section{REFERENCES}

Almenara J. M. et al., 2009, Astronomy and Astrophysics, 506, 337

Alonso R. et al., 2004, The Astrophysical Journal, 613, L153

Armstrong D. J. et al., 2016, Monthly Notices of the Royal Astronomical Society, 456, 2260

Armstrong D. J., Pollacco D., Santerne A., 2017, Monthly Notices of the Royal Astronomical Society, 465, 2634

Bakos G., Noyes R. W., Kovacs G., Stanek K. Z., Sasselov D. D., Domsa I., 2004, Publications of the Astronomical Society of the Pacific, 116, 266

Bakos G. A. et al., 2013, Publications of the Astronomical Society of the Pacific, 125, 154

Bakos G. A. et al., 2007, The Astrophysical Journal, 656, 552

Bayliss D. et al., 2017, Monthly Notices of the Royal Astronomical Society

Blomme J. et al., 2010, The Astrophysical Journal, 713, L204

Borucki W. J. et al., 2010, Science, 327, 977

Bouchy F. et al., 2005, Astronomy and Astrophysics, 444, L15

Brahm R. et al., 2017, eprint arXiv:1707.07093

Breiman L., 2001, Machine Learning, 45, 5

Brett D. R., West R. G., Wheatley P. J., 2004, Monthly Notices of the Royal Astronomical Society, 353, 369

Brink H., Richards J. W., Poznanski D., Bloom J. S., Rice J., Negahban S., Wainwright M., 2013, Monthly Notices of the Royal Astronomical Society, 435, 1047

Cabrera J. et al., 2017, Astronomy and Astrophysics, 606, A75
Carrasco D. et al., 2015, Astronomy and Astrophysics, 584, A44

Carrasco Kind M., Brunner R. J., 2014, Monthly Notices of the Royal Astronomical Society, 438, 3409

Charbonneau D., Brown T. M., Latham D. W., Mayor M., 2000, The Astrophysical Journal, 529, L45

Collier Cameron A. et al., 2007a, Monthly Notices of the Royal Astronomical Society, 375, 951

Collier Cameron A. et al., 2006, Monthly Notices of the Royal Astronomical Society, 373, 799

Collier Cameron A. et al., 2007b, Monthly Notices of the Royal Astronomical Society, 380, 1230

Coughlin J. L. et al., 2016, The Astrophysical Journal Supplement Series, 224, 12

Crossfield I. J. M. et al., 2016, The Astrophysical Journal Supplement Series, 226, 7

Debosscher J., Blomme J., Aerts C., De Ridder J., 2011, Astronomy and Astrophysics, 529, A89

Delrez L. et al., 2014, Astronomy and Astrophysics, 563, A143

Díaz R. F., Almenara J. M., Santerne A., Moutou C., Lethuillier A., Deleuil M., 2014, Monthly Notices of the Royal Astronomical Society, 441, 983

Dittmann J. A. et al., 2017, Nature, 544, 333

Eyer L., Blake C., 2005, Monthly Notices of the Royal Astronomical Society, 358, 30

Farrell S. A., Murphy T., Lo K. K., 2015, The Astrophysical Journal Letters, 813, 28

Fawcett T., 2006, Pattern Recognition Letters, 27, 861

Fressin F. et al., 2013, The Astrophysical Journal, 766, 81

Günther M. N., Queloz D., Demory B.-O., Bouchy F., 2017,

Monthly Notices of the Royal Astronomical Society, 465, 3379

Günther M. N. et al., 2017, Monthly Notices of the Royal Astronomical Society, 472, 295

Hanke M., Halchenko Y. O., Sederberg P. B., Hanson S. J., Haxby J. V., Pollmann S., 2009, Neuroinformatics, 7, 37

Hartman J. D., Bakos G. Á., Torres G., 2011, in European Physical Journal Web of Conferences. p. 2002

Hellier C. et al., 2017, Monthly Notices of the Royal Astronomical Society, 465, 3693

Hellier C. et al., 2014, Monthly Notices of the Royal Astronomical Society, 440, 1982

Huang C., Ma Y.-h., Zhao H.-b., Lu X.-p., 2017, Chinese Astronomy and Astrophysics, 41, 549

Kohonen T., 1982, Biological Cybernetics, 43, 59

Kovacs G., Zucker S., Mazeh T., 2002, Astronomy and Astrophysics, 391, 369

Kreidberg L., 2015, Publications of the Astronomical Society of the Pacific, 127, 1161

Latham D. W. et al., 2009, The Astrophysical Journal, 704, 1107

LeCun Y., Bengio Y., Hinton G., 2015, Nature, 521, 436

Liu C., Deng N., Wang J. T. L., Wang H., 2017, The Astrophysical Journal Letters, 843, 104

Liu F. T., Ting K. M., Zhou Z.-H., 2008, in 2008 Eighth IEEE International Conference on Data Mining (ICDM). IEEE, pp 413-422

McCauliff S. D. et al., 2015, The Astrophysical Journal, 806,6

McCormac J., Pollacco D., Skillen I., Faedi F., Todd I., Watson C. A., 2013, Publications of the Astronomical So- 
ciety of the Pacific, 125,548

McCormac J. et al., 2017, Publications of the Astronomical Society of the Pacific, 129, 025002

McCullough P. R., Stys J. E., Valenti J. A., Fleming S. W., Janes K. A., Heasley J. N., 2005, Publications of the Astronomical Society of the Pacific, 117, 783

Mahabal A. et al., 2008, Astronomische Nachrichten, 329, 288

Mandel K., Agol E., 2002, The Astrophysical Journal, 580, L171

Masci F. J., Hoffman D. I., Grillmair C. J., Cutri R. M., 2014, The Astronomical Journal, 148, 21

Mislis D., Bachelet E., Alsubai K. A., Bramich D. M., Parley N., 2015, Monthly Notices of the Royal Astronomical Society, 455, 626

Morton T. D., 2012, The Astrophysical Journal, 761, 6

Morton T. D., Bryson S. T., Coughlin J. L., Rowe J. F., Ravichandran G., Petigura E. A., Haas M. R., Batalha N. M., 2016, The Astrophysical Journal, 822, 86

Nun I., Pichara K., Protopapas P., Kim D.-W., 2014, The Astrophysical Journal, 793, 23

Pearson K. A., Palafox L., Griffith C. A., 2018, Monthly Notices of the Royal Astronomical Society, 474, 478

Pedregosa F. et al., 2011, Journal of Machine Learning Research, 12, 2825

Pepper J. et al., 2007, Publications of the Astronomical Society of the Pacific, 119, 923

Pollacco D. L. et al., 2006, Publications of the Astronomical Society of the Pacific, 118, 1407

Richards J. W. et al., 2011, The Astrophysical Journal, 733, 10

Richards J. W., Starr D. L., Miller A. A., Bloom J. S., Butler N. R., Brink H., Crellin-Quick A., 2012, The Astrophysical Journal Supplement Series, 203, 32

Ricker G. R. et al., 2014, in SPIE Astronomical Telescopes + Instrumentation. SPIE, p. 914320

Santerne A. et al., 2015, Monthly Notices of the Royal Astronomical Society, 451, 2337

Shallue C. J., Vanderburg A., 2017, eprint arXiv:1712.05044

Shporer A. et al., 2017, The Astrophysical Journal, 847, L18

Smith J. C. et al., 2012, Publications of the Astronomical Society of the Pacific, 124, 1000

Stumpe M. C. et al., 2012, Publications of the Astronomical Society of the Pacific, 124, 985

Sullivan P. W. et al., 2015, The Astrophysical Journal Letters, p. 77

Tamuz O., Mazeh T., Zucker S., 2005, Monthly Notices of the Royal Astronomical Society, 356, 1466

Thompson S. E. et al., 2017, eprint arXiv:1710.06758

Thompson S. E., Mullally F., Coughlin J., Christiansen J. L., Henze C. E., Haas M. R., Burke C. J., 2015, The Astrophysical Journal Letters, p. 46

Torres G. et al., 2015, The Astrophysical Journal, 800, 99 Wheatley P. J. et al., 2017, Monthly Notices of the Royal Astronomical Society 